\section{Nanopore/lllumina Hybrid Genome Sequence Resource for Corynespora cassiicola Strain XJ Infecting Rubber Tree in China}

\author{
Chao Xu, ${ }^{1}$ Caiying Xue, ${ }^{1}$ Mengyuan Hou, ${ }^{1}$ Yuehua Geng, ${ }^{1}$ Rui Zang, ${ }^{1}$ Haiyan $\mathrm{Wu},{ }^{2}$ and \\ Meng Zhang ${ }^{1, \dagger}$ \\ ${ }^{1}$ Department of Plant Pathology, Henan Agricultural University, Zhengzhou 450002, China \\ ${ }^{2}$ Centre for Modern Experimental Technology, Henan Agricultural University, Zhengzhou 450002, \\ China
}

\begin{abstract}
Corynespora cassiicola is a ubiquitous pathogenic fungus that can infect a broad range of plant hosts. Corynespora leaf fall, caused by C. cassiicola, is one of the major diseases on rubber tree in China. This disease is having an increasing affect on natural rubber production worldwide. In this study, by combining the Nanopore and Illumina sequencing technologies, we present the chromosome-scale genome sequence of the rubber treesampled C. cassiicola strain XJ collected in the subtropical region of China. The assembly consists of 23 scaffolds $\left(\mathrm{N}_{50}=4.62 \mathrm{Mb}\right.$ ) with an estimated genome size of $44.42 \mathrm{Mb}$ (only 166 non-ATCG bases) and 16,108 protein-coding genes. The genome will provide a valuable resource for further research on the pathogenesis and comparative genomics of $C$. cassiicola on rubber tree and other hosts.
\end{abstract}

Rubber tree (Hevea brasiliensis (Willd. ex A. Juss.) Müll. Arg.), producing the vast majority of natural rubber, is an economically important tropical rainforest tree native to the Amazon Basin (Sant'Anna et al. 2021). The cash crop was first introduced in Yunnan Province of China in 1904, and then widely cultivated in several southern provinces, including Hainan, Guangxi, and Guangdong (Shi et al. 2019). Due to the extensive application of natural rubber in many related fields (e.g., medical and chemical industries), China has become the world's fifth largest rubber-producing nation and biggest rubber importer (Shi et al. 2019). Recently, however, Corynespora leaf fall (CLF) disease, caused by Corynespora cassiicola (Berk. \& M. A. Curtis) C.T. Wei, is occurring and spreading in most rubber plantations across South China, and severely impairs the yield of rubber (Li et al. 2015). The CLF disease, characterized by leaf necrosis surrounded by yellow halos and premature senescence of young leaves of susceptible cultivars, was first observed in Sierra Leone in 1936. In the late 1980s, after a severe outbreak in Sri Lanka, this disease was regarded as a threat to rubber production (Ribeiro et al. 2019). Since then, CLF has continued to spread to rubber-producing areas in Asia and Africa, gradually developing into one of the most destructive diseases of rubber tree worldwide (Lopez et al. 2018).

In addition to rubber tree, the broad-spectrum ascomycete $C$. cassiicola can also infect more than 500 plant species, including cucumber, tomato, cotton, and soybean. It has even occasionally been isolated from humans (Looi et al. 2017). Genome sequence resources may help explore the molecular mechanisms underlying the wide adaptability (i.e., wide geographic and host ranges) and pathogenicity of $C$. cassiicola. In recent years, although the genome sequencing of three different $C$. cassiicola strains (CCP from rubber tree in the Philippines, UM591 from human in Malaysia, and HGCC from cucumber in China) was successively

${ }^{\dagger}$ Corresponding author: M. Zhang; zm2006@126.com

The author(s) declare no conflict of interest.

Accepted for publication 19 May 2021.
Funding:

This work was supported by the Science and Technology Key Project of Henan Province (192102110164) and Natural Science Foundation of Henan Province (202300410200).

\section{Keywords}

Corynespora cassiicola, genome assembly, leaf fall, Nanopore, rubber tree 
Table 1. Comparison of genome features among the four Corynespora cassiicola strains CM1, XJ, HGCC, CCP, and UM591

\begin{tabular}{|c|c|c|c|c|}
\hline Features & $\mathbf{X J}$ & CCP & UM591 & HGCC \\
\hline Source of strain & Rubber in China & Rubber in Philippines & Human in Malaysia & Cucumber in China \\
\hline Sequencing method & Nanopore/Illumina & Illumina & Illumina & Illumina \\
\hline Genome assembly size (Mb) & 44.42 & 44.85 & 41.88 & 42.70 \\
\hline Number of scaffolds & 23 & 244 & 189 & 1032 \\
\hline Number of gaps & 2 & 256 & - & - \\
\hline Number of non-ATCG bases in gaps & 166 & 142,491 & - & - \\
\hline Scaffold $N_{50}(k b)$ & 4,627 & 2,506 & 1,460 & 500 \\
\hline Scaffold $L_{50}$ & 4 & 8 & - & - \\
\hline Scaffold maximum (kb) & 6,676 & 4,763 & - & - \\
\hline Number of protein-coding genes & 16,108 & 17,167 & 13,531 & 14,631 \\
\hline GC content $(\%)$ & 50.65 & 50.61 & 52.47 & 51.78 \\
\hline Repeated sequences (\%) & 8.58 & 4.88 & - & 0.52 \\
\hline Transfer RNA genes & 151 & 149 & 164 & - \\
\hline Gene density (genes per Mb) & 363 & 383 & 323 & 343 \\
\hline Reference & This work & Lopez et al. 2018 & Looi et al. 2017 & Gao et al. 2020 \\
\hline
\end{tabular}

completed (Gao et al. 2020; Looi et al. 2017; Lopez et al. 2018), ultralong-read sequencing, such as offered by Pacific Biosciences (PacBio) and Oxford Nanopore Technologies (ONT), has never been applied. This approach can improve the quality and integrity of genome assembly. To improve the assembly quality of the rubber tree-sampled C. cassiicola strain XJ from China, we have combined the Nanopore and Illumina sequencing methods.

\section{Genome Sequencing and Assembly}

The $C$. cassiicola strain $\mathrm{XJ}$ was obtained by single-spore isolation from an infected leaf of rubber tree in Yunnan, China (22.00 N 100.77 E) in 2016, and is now preserved in the fungal collection of Henan Agricultural University. This strain was grown on sterile potato dextrose agar at $25^{\circ} \mathrm{C}$ in the dark for 5 days before mycelium was harvested and ground with liquid nitrogen. Genomic DNA was extracted from the fresh mycelium using a modified cetyltrimethylammonium bromide method (Murray and Thompson 1980). Whole-genome sequencing was performed on an Oxford Nanopore MinION platform and an Illumina HiSeq X Ten platform (Sino Genomics Co., Ltd., Shenzhen, China). For Nanopore sequencing, 4.4 Gb of FASTQ data were obtained by format conversion of the raw FAST5 files using Albacore v2.2.7 (https://github.com/ Albacore/albacore). Adapter sequences and low-quality reads (average quality $<$ Q8) were then filtered out using Porechop v0.2.3 (https://github.com/rrwick/Porechop) and NanoFilt v2.2.0 (De Coster et al. 2018), respectively. Multiple rounds of error corrections were run by Canu v1.7 (Koren et al. 2017). For Illumina sequencing, the raw data (6.9 Gb) were trimmed of adapters and low-quality reads (ratio of the bases less than Q20 $>30 \%$ or containing undetermined bases) by using fastp v0.13.1 (Chen et al. 2018). In total, $4.1 \mathrm{~Gb}$ of Nanopore clean data (876,932 long reads with maximum length of $133,489 \mathrm{bp}$ and read $\mathrm{N}_{50}$ of $6,735 \mathrm{bp}$ ) and $5.7 \mathrm{~Gb}$ of Illumina clean data $(38,000,844$ paired-end reads with the same length of $150 \mathrm{bp})$ were obtained. This corresponded to a depth of approximately 89-fold and approximately 125 -fold for the MinION and Illumina data, respectively. Following a hybrid Nanopore/lllumina approach, both the above cleaned long and short reads were de novo assembled using MaSuRCA v3.2.6 (Zimin et al. 2013) with the default parameters. Then, the raw assembly was further polished with the Illumina short reads using Pilon v1.22 (Walker et al. 2014), correcting 3,398 mismatches in total, including 829 single-nucleotide polymorphisms (SNPs) and 2,569 insertion/deletions (indels). The final genome assembly of $C$. cassiicola strain $\mathrm{XJ}$ comprised 23 scaffolds with a total size of $44.42 \mathrm{Mb}$ and an $\mathrm{N}_{50}$ of $4.63 \mathrm{Mb}$. In all, 166 ambiguous (nonATCG) positions, forming two gaps, were detected in this chromosome-scale assembly. The GC content was $50.7 \%$. To evaluate the quality of the genome assembly, the Illumina short reads and Nanopore long reads were mapped using BWA v0.7.17 (Li and Durbin 2009) and GraphMap v0.5.2 (Sović et al. 2016), which revealed genome mapping ratios of 97.37 and 94 . $17 \%$, respectively. Completeness of the genome was assessed by using Benchmarking Universal Single-Copy Orthologs (BUSCOs) v3.0.1 (Simão et al. 2015) against the fungi_odb10 dataset. This analysis revealed that $99.5 \%$ of the 758 fungal BUSCO groups (including $99.1 \%$ complete single-copy BUSCOs and $0.4 \%$ complete duplicated BUSCOs) were identified in the C. cassiicola strain $\mathrm{XJ}$ genome, indicating a high level of completeness. 


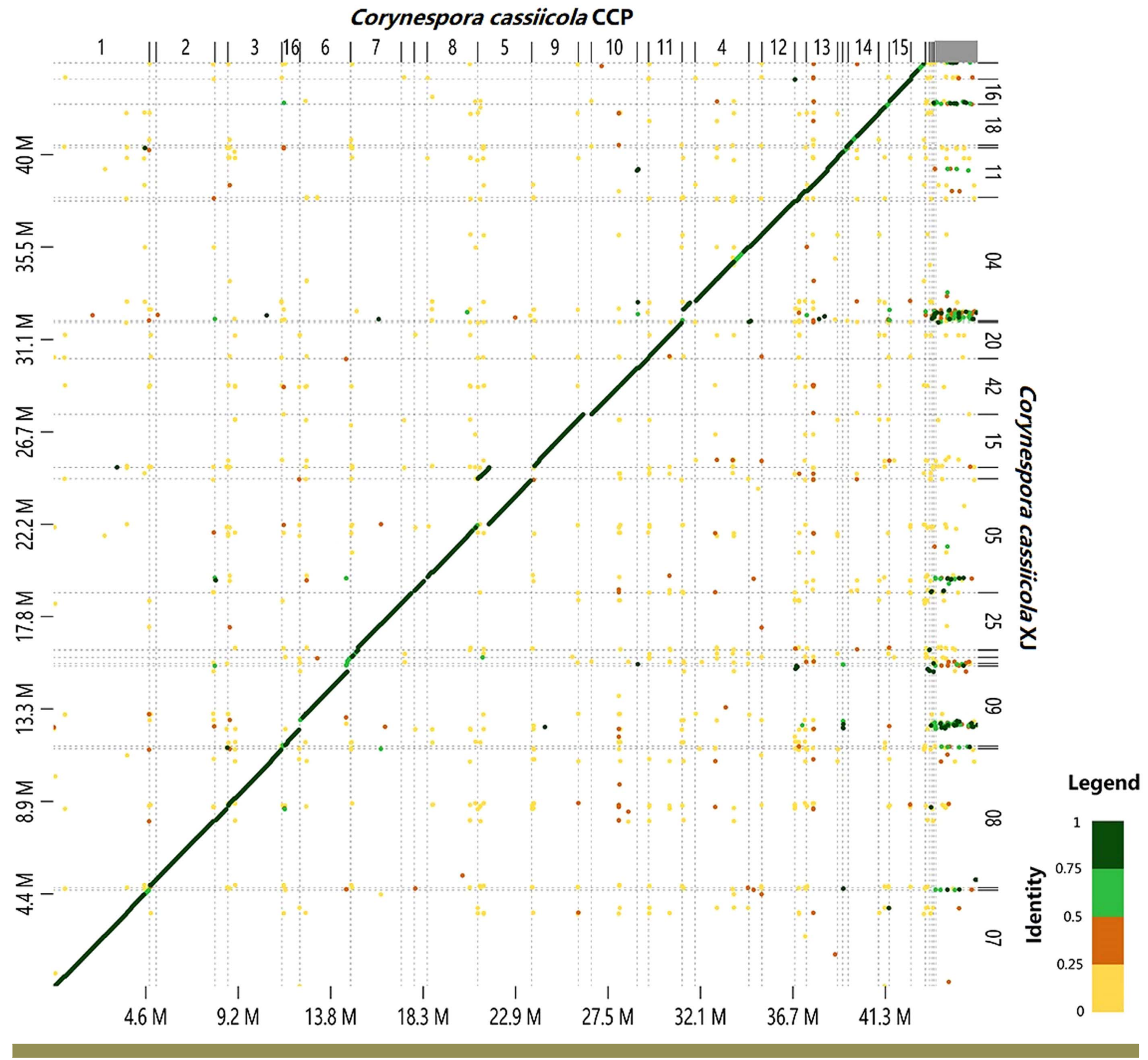

Fig. 1. Synteny dot plot of the genomic scaffolds between Corynespora cassiicola strains CCP and XJ. CCP as a target is on the X-axis and XJ as a query is on the $y$-axis. Gridlines demarcate scaffolds. Genomic alignment regions are presented as four-colored lines, corresponding to different similarity values: $<25,25$ to 50,50 to 75 , and $>75 \%$.

Compared with the previously assembled $C$. cassiicola genome sequence of strain $\mathrm{CCP}$ (Lopez et al. 2018), the genome assembly of strain XJ has fewer scaffolds (244 versus 23), a larger $\mathrm{N}_{50}$ value (2.51 versus $\left.4.63 \mathrm{Mb}\right)$, and a much smaller non-ATCG rate (3.18e-03 versus 3.74e-06) (Table 1). This evidence suggests that the genome of strain $\mathrm{XJ}$ is more complete and of higher quality than previously reported genomes of this pathogen. In addition, whole-genome comparisons using MUMmer v3.23 (Kurtz et al. 2004) between the XJ and CCP strains (both isolated from rubber tree) suggested a high-level dissimilarity (approximately 1 SNP per 43 bp). Their synteny analysis using D-GENIES (Cabanettes and Klopp 2018) revealed a few chromosomal rearrangements, principally large indels and translocations (Fig. 1), and that only approximately $80 \%$ of the CCP genome assembly showed over $75 \%$ sequence identity with XJ. These results support the hypothesis that the high degree of genetic variation within the species is correlated with geographic isolation (Dixon et al. 2009).

\section{Genome Annotation}

The transfer RNA (tRNA) and its secondary structure were predicted by tRNAscan-SE v2.0 (Chan and Lowe 2019), identifying 151 tRNA genes. Repetitive elements were detected and 
annotated using RepeatMasker v4.1.0 with both the RepBase-20170127 fungal library and ab initio libraries generated by RepeatModeler (http://www.repeatmasker.org/). In total, 3,809,464 bp of repeat sequences were detected in $8.58 \%$ of the genome assembly. High-quality gene calling was performed using the MAKER2 pipeline v2.31.10 (Holt and Yandell 2011), which combines three different ab initio predictors-GeneMark-ES (Ter-Hovhannisyan et al. 2008), Augustus (Stanke et al. 2006), and SNAP (Korf 2004)—and utilizes the homology-based gene predictor Exonerate v2.2.0 and 8.66 Gb of RNA-sequencing data (cleaned Illumina paired-end reads) to help improve gene structural annotations. Overall, 16,108 protein-coding regions were predicted in the $\mathrm{XJ}$ genome, and were then functionally assigned by BLASTp searches against some comprehensive and specialized databases. Across all gene models, approximately 95 . 23, 53.40, 42.02, 25.02, and 64.54\% were mapped in the databases of nonredundant protein sequences, SwissProt, Eukaryotic Orthologous Groups, Kyoto Encyclopedia of Genes and Genomes, and Integrated Resource of Protein Domains and Functional Sites, respectively. Furthermore, in total, 852 transporter proteins, 878 peptidases, 600 carbohydrate active enzymes, and 640 cytochrome P450s were successfully predicted against the Transporter Classification Database, MEROPS (release 12.3), dbCAN2, and Cytochrome P450 Engineering Database v6.0 databases.

In view of the pathogenicity of $C$. cassiicola, we performed BLASTP similarity searches against the pathogen-host interaction database (http://www.phi-base.org) and identified 4,644 potential virulence-related genes within the XJ genome. Additionally, several specific categories of pathogenicity-related genes such as plant cell-wall-degrading enzymes (PCDWE), secondary metabolite synthesis backbone enzymes (SMBE), and candidate-secreted effector proteins (CSEP) were manually annotated (Xu et al. 2016, 2017). In total, 226 putative PCDWE were identified, corresponding to 178 glycoside hydrolases, 38 polysaccharide lyases, and 10 carbohydrate esterases. In total, 58 SMBEs, including 30 polyketide synthases, 18 nonribosomal peptide synthetases, 2 dimethylallyl tryptophan synthases, and 8 terpene cyclases, were identified. Approximately $5.3 \%$ of the total proteins of $C$. cassiicola strain XJ were predicted to be secreted, of which 117 were identified as cysteine-rich CSEP.

In the present study, we provide a chromosome-scale genomic assembly of $C$. cassiicola strain XJ infecting rubber tree in Southern China. This resource will be useful for future studies on the mechanisms of virulence, population genetics, and disease resistance for this economically important fungal pathogen.

\section{Data Availability}

The whole-genome data of $C$. cassiicola strain XJ presented here has been deposited at GenBank under the accession number JAEUWY000000000 (BioProject PRJNA694745; BioSample SAMN17573700).

\section{Literature Cited}

Cabanettes, F., and Klopp, C. 2018. D-GENIES: Dot plot large genomes in an interactive, efficient and simple way. PeerJ 6:e4958.

Chan, P. P., and Lowe, T. M. 2019. tRNAscan-SE: Searching for tRNA genes in genomic sequences. Methods Mol. Biol. 1962:1-14.

Chen, S., Zhou, Y., Chen, Y., and Gu, J. 2018. Fastp: An ultra-fast all-in-one FASTQ preprocessor. Bioinformatics 34:i884-i890.

De Coster, W., D'Hert, S., Schultz, D. T., Cruts, M., and Van Broeckhoven, C. 2018. NanoPack: Visualizing and processing long-read sequencing data. Bioinformatics 34:2666-2669.

Dixon, L. J., Schlub, R. L., Pernezny, K., and Datnoff, L. E. 2009. Host specialization and phylogenetic diversity of Corynespora cassiicola. Phytopathology 99:10151027.

Gao, S., Zeng, R., Xu, L., Song, Z., Gao, P., and Dai, F. 2020. Genome sequence and spore germination associated transcriptome analysis of Corynespora cassiicola from cucumber. BMC Microbiol. 20:199.

Holt, C., and Yandell, M. 2011. MAKER2: An annotation pipeline and genomedatabase management tool for second-generation genome projects. BMC Bioinf. 12:491.

Koren, S., Walenz, B. P., Berlin, K., Miller, J. R., Bergman, N. H., and Phillippy, A. M. 2017. Canu: Scalable and accurate long-read assembly via adaptive k-mer weighting and repeat separation. Genome Res. 27:722-736.
Korf, I. 2004. Gene finding in novel genomes. BMC Bioinf. 5:59.

Kurtz, S., Phillippy, A. M., Delcher, A. L., Smoot, M. E., Shumway, M., Antonescu, C., and Salzberg, S. L. 2004. Versatile and open software for comparing large genomes. Genome Biol. 5:R12.

Li, B., Liu, X., Cai, J., and Huang, G. 2015. Investigation and occurrence regularity of Corynespora defoliation diseases of rubber tree in China. Chin. J. Trop. Crops 36:2058-2066.

Li, H., and Durbin, R. 2009. Fast and accurate short read alignment with BurrowsWheeler transform. Bioinformatics 25:1754-1760.

Looi, H. K., Toh, Y. F., Yew, S. M., Na, S. L., Tan, Y. C., Chong, P. S., Khoo, J. S., Yee, W. Y., Ng, K. P., and Kuan, C. S. 2017. Genomic insight into pathogenicity of dematiaceous fungus Corynespora cassiicola. PeerJ 5:e2841.

Lopez, D., Ribeiro, S., Label, P., Fumanal, B., Venisse, J. S., Kohler, A., de Oliveira, R. R., Labutti, K., Lipzen, A., Lail, K., Bauer, D., Ohm, R. A., Barry, K. W., Spatafora, J., Grigoriev, I. V., Martin, F. M., and Pujade-Renaud, V. 2018. Genome-wide analysis of Corynespora cassiicola leaf fall disease putative effectors. Front. Microbiol. 9:276.

Murray, M. G., and Thompson, W. F. 1980. Rapid isolation of high molecular weight plant DNA. Nucleic Acids Res. 8:4321-4326.

Ribeiro, S., Tran, D. M., Déon, M., Clément-Demange, A., Garcia, D., Soumahoro, M., Masson, A., and Pujade-Renaud, V. 2019. Gene deletion of Corynespora cassiicola cassiicolin Cas1 suppresses virulence in the rubber tree. Fungal Genet. Biol. 129:101-114. 
Sant'Anna, I. de C., Gouvêa, L. R. L., Martins, M. A., Saloppi Junior, E. J., de Freitas, R. S., and de Souza Gonçalves, P. 2021. Genetic diversity associated with natural rubber quality in elite genotypes of the rubber tree. Sci. Rep. 11:1081.

Shi, T., Li, B., Feng, Y., Liu, X., Zheng, X., and Huang, G. 2019. Safety assessment of Corynespora leaf fall disease on natural rubber and related industries in China. Chin. J. Trop. Agric. 39:66-71.

Simão, F. A., Waterhouse, R. M., loannidis, P., Kriventseva, E. V., and Zdobnov, E. M. 2015. BUSCO: Assessing genome assembly and annotation completeness with single-copy orthologs. Bioinformatics 31:3210-3212.

Sović, I., Šikić, M., Wilm, A., Fenlon, S. N., Chen, S., and Nagarajan, N. 2016. Fast and sensitive mapping of nanopore sequencing reads with GraphMap. Nat. Commun. 7:11307.

Stanke, M., Keller, O., Gunduz, I., Hayes, A., Waack, S., and Morgenstern, B. 2006. AUGUSTUS: $A b$ initio prediction of alternative transcripts. Nucleic Acids Res. 34:W435-W439.
Ter-Hovhannisyan, V., Lomsadze, A., Chernoff, Y. O., and Borodovsky, M. 2008. Gene prediction in novel fungal genomes using an ab initio algorithm with unsupervised training. Genome Res. 18:1979-1990.

Walker, B. J., Abeel, T., Shea, T., Priest, M., Abouelliel, A., Sakthikumar, S., Cuomo, C. A., Zeng, Q., Wortman, J., Young, S. K., and Earl, A. M. 2014. Pilon: An integrated tool for comprehensive microbial variant detection and genome assembly improvement. PLoS One 9:e112963.

Xu, C., Chen, H., Gleason, M. L., Xu, J., Liu, H., Zhang, R., and Sun, G. 2016 Peltaster fructicola genome reveals evolution from an invasive phytopathogen to an ectophytic parasite. Sci. Rep. 6:22926.

Xu, C., Zhang, R., Sun, G., and Gleason, M. L. 2017. Comparative genome analysis reveals adaptation to the ectophytic lifestyle of sooty blotch and flyspeck fungi. Genome Biol. Evol. 9:3137-3151.

Zimin, A. V., Marçais, G., Puiu, D., Roberts, M., Salzberg, S. L., and Yorke, J. A. 2013. The MaSuRCA genome assembler. Bioinformatics 29:2669-2677. 